\author{
22-24 November, $2019 \quad$ Paris, France
}

\title{
Preparation and Physicochemical Properties of Nano porous Mgo Using Micro Emulsion Technique
}

\author{
Atthaphon Maneedaeng', Panuwat Lawtae ${ }^{2}$, Arissara Boonma ${ }^{3}$, Wasupol Buathonglang ${ }^{4}$ \\ School of Chemical Engineering, Institute of Engineering, Suranaree University of Technology, Nakhon \\ Ratchasima, 30000 Thailand
}

\begin{abstract}
Various nanoscaled pore diameters of $\mathrm{MgO}$ have been prepared via surfactant-1-butanol-isooctanenitrate-oxalate salt microemulsion. The surfactants of AOT and Dehydol LS3 TH were used in this work in order to compare the difference in porous structures. Physicochemical properties of the prepared nanoscaled pore diameters of $\mathrm{MgO}$ were characterized by Zetasizer, X-ray diffraction, UV-Vis Spectrometer, Scanning Electron Microscope, and Brunauer-Emmett-Teller (BET). The experimental results showed that average particle size of $\mathrm{MgO}$ prepared in microemulsion with AOT was smaller than in Dehydol LS3 TH, and higher calcined temperature resulted in smaller average particle size. The XRD patterns indicated that all surfactants were removed during calcination and all $\mathrm{MgO}$ produced by this technique was pure. The maximum absorptions were determined at 215-218 nm with the electron band gaps of 5.70-5.81 eV. The SEM images showed that the surfactants used resulted in different morphology and nanoporous structure. The results from BET revealed that both anionic and nonionic surfactants effected on $\mathrm{N}_{2}$ adsorption and desorption. The differences in results could be due to the reverse micellization in microemulsion technique. Besides, the difference in calcined temperature of $600-800{ }^{\circ} \mathrm{C}$ resulted in decreasing the BET surface area and pore size when temperature increased.
\end{abstract}

Keywords: Magnesium Oxide; Reverse Micellar System; Microporous Materials; Adsorption 